\title{
Supercurrents at a pinch
}

\section{John Gallop}

IF ANY one property is expected of a superconductor it is that it should carry high currents without resistive loss. In the case of the high-temperature superconductors the promise of their dramatically increased transition temperatures (up to $125 \mathrm{~K}$, compared with $23 \mathrm{~K}$ for the best conventional superconductors) was initially tempered by the experimental observation of rather low critical currents, that is the maximum value passed before dissipation becomes detectable. But in Physical Review Letters last week (66, 1785-1788; 1991), W. Jiang et al. amply confirmed what many workers in the field had come to believe: that it will not be the intrinsic current carrying capabilities of the high-temperature materials that will prevent their widespread technological application. The authors report that reducing the width of the currentcarrying channel dramatically increases a superconductor's currentcarrying capability, as characterized by the critical current density.

Jiang et al. set out to see the effect of passing a supercurrent through a narrow constriction in thin films of $\mathrm{YBa}_{2} \mathrm{Cu}_{3} \mathrm{O}_{7}$, the ubiquitous hightemperature superconductor with a

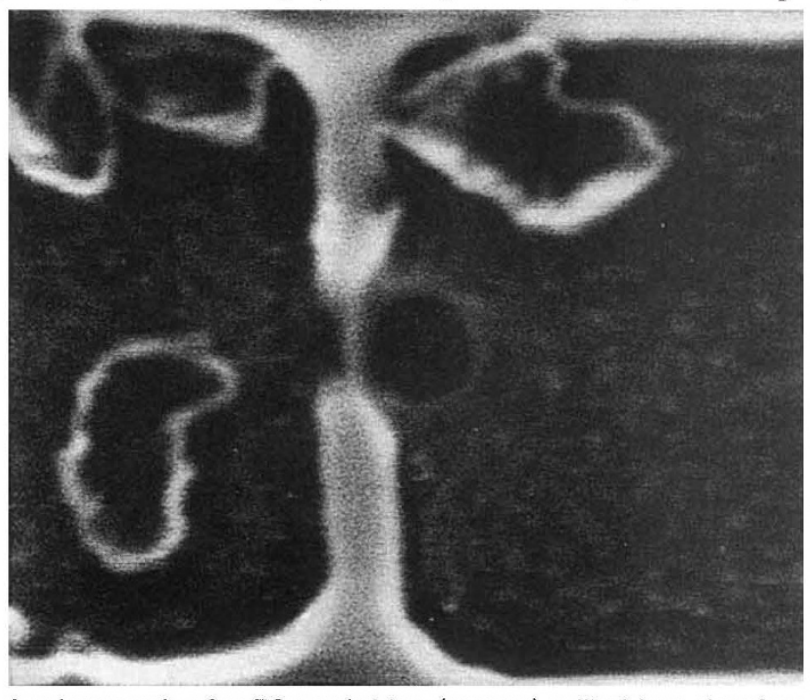

pinning flux. But it has been recognized for many years that another approach, not obviously of practical use, may also be followed. By making the cross-sectional area of the conductor smaller one may exploit an energy barrier which grows to prevent fluxons entering the conducting channel, and this is the approach taken by Jiang et al. The exclusion of fluxons from the bridge then removes the restriction on critical current due to fluxon flow, thereby allowing the depairing limit to be approached.

The characteristic lengths $\lambda$ and $\xi$, which determine the depairing limit, are somewhat sample dependent and difficult to measure; more seriously, they are highly anisotropic in the layered cuprate superconductors. The highest value of $j_{\mathrm{c}}$ that Jiang et al. measure, $1.3 \times 10^{9} \mathrm{~A} \mathrm{~cm}^{-2}$, is a factor of 10 higher than has been previously reported for any other superconductor, whether conventional or cuprate; but it is still less than the depairing limit calculated using reasonable values of the characteristic lengths (with $\lambda=100 \mathrm{~nm}$ and $\xi=1 \mathrm{~nm}$, one expects $j_{\mathrm{c}}=3 \times 10^{10} \mathrm{~A} \mathrm{~cm}^{-2}$ ).

Because $j_{\mathrm{c}}$ continued to increase even when the width of the bridge had been reduced to $50 \mathrm{~nm}$, Jiang et al. propose that another dissipation mode is operating. This involves the generation of fluxons in a fashion analogous to the process that creates transition temperature $T_{\mathrm{c}}$ of $91 \mathrm{~K}$. A micrograph of a 50-nm bridge (centre) milled into thin-film vortices in superfluid helium, the First, a coarse pattern was chemically $\mathrm{YBa}_{2} \mathrm{Cu}_{3} \mathrm{O}_{7}$, giving superior current-carrying characteristics. Onsager-Feynman process. (R. $P$. etched in films 200-500 nm thick, (Courtesy, A. Widom.)

before a finely focused beam of gallium ions was used to mill narrow bridges $50-400 \mathrm{~nm}$ across (see micrograph). The authors found that reducing the width of the bridge increased the critical current density the material could carry. Even at a width of $50 \mathrm{~nm}$, the critical current $j_{\mathrm{c}}$ had not reached a plateau, contrary to expectations, causing the authors to put forward a new model for the current limit.

Several mechanisms are known to limit the critical current of a superconducting filament. Perhaps the most fundamental is the critical 'depairing' current density: superconductivity occurs when electrons 'condense' into loosely bound pairs, and the depairing $j_{\mathrm{c}}$ can be understood simply as that value of current for which the local kinetic energy density of the pairs becomes greater than the condensation energy density. The latter is simply related to the thermodynamic critical magnetic field $H_{\mathrm{c}}$, which also suppresses superconductivity. The relatively low values of the depairing $j_{\mathrm{c}}$ found in elemental superconductors such as tin or indium agree approximately with this simple model which links the low-critical fields for such materials with their limited ability to carry current.

In some so-called type II superconductors, to which category belong all of the high- $T_{\mathrm{c}}$ cuprates so far investigated, there is another quenched and the quantized fluxon threads this region. This core is surrounded by a region of circulating supercurrent which extends over an annular length $\lambda$, the penetration depth of the superconductor.

The ability of a type II superconductor to remain lossless in the regions between the normal fluxon cores is necessary but not sufficient for high-current or high-field applications. One extra trick is needed: to fix the lattice of fluxons. In the presence of a current through the superconductor these miniature magnets experience a force at right angles to both the direction of current and to the direction of the flux quantum (as a result of Ampere's law). Motion of the fluxons produces a voltage by induction along the superconductor, with resulting dissipation. In an ideal material this flux flow would occur at arbitrarily low current densities. In real materials, fluxons are pinned by defects and impurities, with no movement allowed until the driving force, proportional to current density, can overcome the pinning forces on fluxons. The critical current density arising from this source of dissipation is a function of the strength of the pinning and also of temperature.

To achieve practical high current densities in bulk samples of superconductor the main thrust has been to introduce inhomogeneities in the crystal lattice which are effective in
Feynman Prog. Low-Temp. Phys. 1, $17-53 ; 1955)$. Although the presence of fluxons in a small bridge is energetically forbidden, the current density may be sufficiently high to generate fluxon rings (or more likely, in the highly anisotropic cuprate materials, a pair of oppositely directed fluxons) around the exit of the bridge, where it joins the electrode, rather as a smoker blows smoke rings. The critical current density determined this way depends on the usual two characteristic lengths, but it also varies with the width of the bridge.

Does this result have practical implications? The short, ultrafine superconducting tracks produced in the experiments seem to have little in common with the vast lengths of rugged, unbroken, high-quality conductor which would be required for magnets or power transmission. However, one interesting potential use of the new materials, and one of the most demanding in terms of the critical current density required, is to provide interconnects in very fast cryogenically cooled chips. The demonstrated ability to produce a $50-\mathrm{nm}$-wide track capable of carrying a current of $100 \mathrm{~mA}$ without dissipation might prove extremely useful in this context.

John Gallop is at the National Physical Laboratory, Queens Road, Teddington TW11 OLW, UK. 